\title{
Introduction to the ATLAS detector at the LHC
}

\author{
Ang $L i^{1, a}$ \\ ${ }^{1}$ School of Physics, Shandong University, Jinan 250100, China. \\ a493568352@qq.com
}

Keywords: ATLAS, detector, inner detector, calorimeter, Muon spectrometer.

\begin{abstract}
ATLAS(A Toroidal LHC ApparatuS) is one particle detector at the large hadron collide (LHC), which is the world's largest and most powerful particle accelerator at the EUropean Organization For Nuclear Research(CERN) in Switzerland. ATLAS is intended to be a general-purpose detector, rather than focusing on a particular physical process. The most famous result on ATLAS is that it (together with CMS, another detector at LHC) reported the evidence for the existence of a particle consistent with the Higgs boson. The paper is a survey of the component and mechanism of the ATLAS detector. The ATLAS detector consists of three major parts: the Inner Detector, the Calorimeter, the Muon Spectrometer. The Inner Detector consists of the pixel detector, the semi-conductor tracker (SCT) and the transition radiation tracker(TRT). The calorimeter consists of electromagnetic calorimeter and the hadron calorimeter. The muon spectrometer is an extremely large tracking system which consists of two major parts: 1) precision-tracking chambers, 2) triggering-tracking chambers.
\end{abstract}

\section{Background}

The Large Hadron Collider (LHC) is the world's largest and most powerful particle collide in the world. It was built by the European Organization for Nuclear Research (CERN) between 1998 and 2008 in collaboration with over 10,000 scientists and engineers from over 100 countries, as well as hundreds of universities and laboratories [1]. It is designed to research the frontier of the particle physics with its high energy and luminosity. The LHC will not only collide protons, but also collide heavy ions. As one of the general purpose detectors, ATLAS can detect p-p and A-A collision. Higgs boson has been detected by CMS and ATLAS in 2012 [3, 4]. Now the energy of the LHC has been improved to $14 \mathrm{TeV}$ so more exciting things such as physics beyond the Standard Model and properties of the Higgs boson might be detected by ATLAS. People may want to know what structure of ATLAS makes it such efficient to detect the particles in collision. This paper give a detail introduction of the structure, component, mechanism and physical program of ATLAS detector.

\section{The Component of ATLAS}

ATLAS is 44 meters long and 25 meters in diameter. It consists of three major parts: the Inner Detector, the Calorimeter, the Muon Spectrometer [2].

The Inner Detector is a track detector which is closest to the proton beam axis. It is immersed in a solenoidal field. The basic functions of Inner Detector are detecting the particles' track and momentum. Through tracking charged particles by detecting their interaction with discrete a high-resolution semiconductor pixel, revealing the types of particles and their momentum.

The calorimeters are situated middle layer which consists of electromagnetic calorimeter and the hadron calorimeter. The electromagnetic (EM) calorimeter can measure the energy of electron and photon. The electron and photon showers happens in the electromagnetic calorimeter. The EM calorimeter absorb the particles' energy and make them slow down and electrons will leave trajectories. The hadron calorimeter can measure the energy of hadron. Proton and neutron showers happen in the hadron calorimeter. When proton and neutron go through the hadron calorimeter and before the showers, proton would leave trajectories but neutron would not. Moreover, muon would go through the hadron calorimeter and leave trajectories. 
The Muon spectrometer locate at the outer layer, which is designed to detect charged particles exiting the calorimeters and to measure their momentum. Muon momenta down to a few $\mathrm{GeV}$ may be measured by the spectrometer alone.

Inner Detector. The Inner Detector is contained in a cylindrical envelope whose inner surface is $50.5 \mathrm{~mm}$ and outer surface is $1150 \mathrm{~mm}$ from the proton beam axis. It length is $\pm 3512 \mathrm{~mm}$. The whole detector locate in solenoidal magnetic field of $2 \mathrm{~T}$ [2]. It is consists of the pixel detector, the semi-conductor tracker (SCT) and the transition radiation tracker (TRT) from inside to outside. In the cylindrical structure, the pixel detector contains three cylindrical layers, the pixel of each of them is $50 \mu \mathrm{m} \times 400 \mu \mathrm{m}$. The SCT contains four cylindrical double layers.

The TRT has 36 straws of $4 \mathrm{~mm}$ diameter. Overall, in the end-cap structure of Inner Detector, the inner part is three pixel layers, the middle part is four double layers of end-cap SCT, the outer part is 36 straws of $4 \mathrm{~mm}$ diameter. Since the closer to the proton beam axis, the denser to the particles, the detector need more precision. Therefore, the PIXEL is the most precision and precision of TRT is relatively lower.

Calorimeter. The calorimeter has a diameter of $12 \mathrm{~m}$ and a length of $4.25 \mathrm{~m}$. The calorimeters closest to the beam-line are housed in three calorimeter, one electromagnetic barrel calorimeter, two end-cap calorimeters (EMEC), hadronic end-cap calorimeters (HEC) and forward calorimeters (FCal). The active detector medium of all these calorimeters are liquid argon. Liquid argon has intrinsic linear behavior, stability of response over time and intrinsic radiation-hardness.

The precision electromagnetic calorimeters have accordion shape absorbers and electrodes, in order to reduce the blind zone. The active detector medium is liquid argon and the absorber is lead. On both side of the lead, using steel to gain in strength. The readout electrodes lie between the absorbers and consist of three conductive copper layers separated by insulating polyimide sheets.

The outer hadronic calorimeter consists of the detector medium and the absorber. But for each hadronic calorimeter, their detector medium and absorber are different. The cylindrical hadronic calorimeter locate outside which has central barrel with $5.8 \mathrm{~m}$ length and two extended barrels with $2.6 \mathrm{~m}$ length. Their detector medium is scintillator and absorber is steel. The edges of tiles are combined with wavelength-shifting fiber readout, and these fibers are connected with photomultiplier tubes (PMT). The end-cap hadronic calorimeter use liquid argon as detector medium and copper as absorber. The forward hadronic calorimeters use liquid argon as detector medium and copper-tungsten as absorber.

Muon Spectrometer. In order to get the precision momentum, the Muon Spectrometer need have large volume. The detector starts at a radius of $4.25 \mathrm{~m}$ out to $11 \mathrm{~m}$, i.e., the full radius of the detector. The distance between the outermost layer of the endcap and the beam axis is about $21 \mathrm{~m}$. The muon spectrometer consists of two major parts: 1) precision-tracking chambers, 2) triggering-tracking chambers. Specifically, precision-tracking chambers contains Monitored drift tube chambers (MDT), cathode-strip chambers (CSC). Triggering-tracking chambers contains Resistive Plate Chambers (RPC), Thin Gap Chambers (TGC). The MDT locates in both the barrel and end-cap region. The CSC is in the end-cap region. The RPC is in the barrel and the TGC is in the end-cap region.

\section{Detection Mechanism}

The Inner Detector consists of the pixel detector (PIXEL), the semi-conductor tracker (SCT) and the transition radiation tracker (TRT). They are used to measure the particles' trajectories. In the PIXEL, the top layer is made of silicon with sensor bias voltage. It connects with a lower layer of readout chip. As a charged particle goes by, it liberate electrons. Then these electrons move to the bottom of the sensor because of the voltage. As a result, an electron current is created. Through the location of the electricity, we can compute the location of particles. Since the PIXEL cover the photon beam. We can ensure every particle's trajectory. SCT is similar to the PIXEL but has lower detection precision. TRT is used to measure the type of the particles. TRT consists of tubes which are full of gas. These tubes has nice electrical and mechanical properties. The anodes of the tubes are made of $31 \mu \mathrm{m}$ tungsten (99.95\%) which is plated with $0.5-0.7 \mu \mathrm{m}$ gold. The cathodes of the tubes is 
situated in mixture gas with 70\%Xe, 27\%CO2 and 3\%O2 [2]. When the charged particles go through the tubes, it generates photons. But the observed results are different for pion and electron. The electron generates more photons. When the photons interactive with the gas molecule, it generates electrons. The process is positive feedback. Therefore, the anodes would detect more negative charge. Thus, TRT can distinguish the pion and electron. Overall, the Inner Detector can measure the particles' trajectories, types and momentums.

The calorimeter is used to measure the particles' energy. The electromagnetic calorimeter is used to measure the energy of electron and photon. Since electron and photon would generate shower which is consist of electrons with lower energy, positron and photon in the detector medium. The low energy shower ionize the medium and generate positive ions and electrons. The electron can be measured by the electrodes made by copper. In order to measure all the electrons and photons, the electromagnetic calorimeter should be large enough. The hadronic calorimeter is used to measure the energy of hadron. Scintillator shall flash when charged particles go through. When high energy hadron pass through steel, it shall interact with nucleus and generates more particles and other further interactions will happen. The program generates shower. The shower make the scintillator transmit radiate light. The light is transformed to electrical signal when collected by fiber and leaded to photomuitplier. Through measuring the electrical signal, we know the hadron's energy. Because, the energy of hadron is much stronger than electron or photon, the size of hadronic calorimeter need be much larger. Overall, we can measure the energy of all particles.

Muon Spectrometer is used to measure the muon's momentum and trajectory. The MDT and CSC is used for precision-tracking. MDT make up most of Muon spectrometer. It is full of AR/CO2 gas. The charged particle shall ionize the gas and generate electrons. The electrons shall accumulate on the tungsten-rhenium wire. We can measure the signal. Through measure the time when electron reach the anodes, we can calculate the momentum and trajectory of the particle.

\section{Conclusion}

The paper introduce the structure, component and mechanism of ATLAS detector. ATLAS consists of inner detector, calorimeter, muon spectrometer from inside to outside. The particles generated by the p-p collision go through the inner detector first. The PIXEL and SCT can measure the particles trajectories and momentums. The TRT can measure the type of the particles. Then the particles go through the calorimeter. The electromagnetic calorimeter measures the electromagnetic energy. The hadronic calorimeter measures the energy of hadrons. The muon and neutrino then pass through muon spectrometer which can measure the trajectory and momentum of muon.

\section{References}

[1] The Large Hadron Collider

[2] Aad, Georges, et al. "The ATLAS experiment at the CERN large hadron collider." Journal of Instrumentation 3.8 (2008).

[3] Aad, Georges, et al. "Observation of a new particle in the search for the Standard Model Higgs boson with the ATLAS detector at the LHC." Physics Letters B 716.1 (2012): 1-29.

[4] Off, CERN Press. "CERN experiments observe particle consistent with long-sought Higgs boson." (2012).

[5] Aad, Georges, et al. "Measurement of the top quark-pair production cross section with ATLAS in pp collisions at sqrt s= 7 TeV." The European Physical Journal C 71.3 (2011): 1-36.

[6] Tuertscher, Philipp, Raghu Garud, and Arun Kumaraswamy. "Justification and interlaced knowledge at ATLAS, CERN." Organization Science 25.6 (2014): 1579-1608. 